\title{
BMI1 maintains the Treg epigenomic landscape to prevent inflammatory bowel disease
}

\author{
Michelle M. Gonzalez, ${ }^{1}$ Adebowale O. Bamidele, ${ }^{1}$ Phyllis A. Svingen, ${ }^{1}$ Mary R. Sagstetter, ${ }^{1}$ Thomas C. Smyrk, ${ }^{2}$ Joseph M. Gaballa, ${ }^{1}$ \\ Feda H. Hamdan, ${ }^{1}$ Robyn Laura Kosinsky, ${ }^{1}$ Hunter R. Gibbons, ${ }^{1}$ Zhifu Sun, ${ }^{3}$ Zhenqing Ye, ${ }^{3}$ Asha Nair, ${ }^{3}$ Guilherme P. Ramos, ${ }^{1}$ \\ Manuel B. Braga Neto, ${ }^{1}$ Alexander Q. Wixom, ${ }^{1}$ Angela J. Mathison, ${ }^{4}$ Steven A. Johnsen, ${ }^{1}$ Raul Urrutia, ${ }^{4}$ and William A. Faubion Jr. ${ }^{1}$ \\ 'Epigenetics and Chromatin Dynamics Laboratory, Division of Gastroenterology and Hepatology and Translational Epigenomic Program, Center for Individualized Medicine (CIM), ²Department of \\ Laboratory Medicine and Pathology, ${ }^{3}$ Department of Genetics and Bioinformatics, Mayo Clinic, Rochester, Minnesota, USA. ${ }^{4}$ Cenomic Sciences and Precision Medicine Center, Medical College of \\ Wisconsin, Milwaukee, Wisconsin, USA.
}

\begin{abstract}
FOXP3+ Tregs are expanded within the inflamed intestine of human Crohn's disease, yet FOXP3-mediated gene repression within these cells is lost. The polycomb repressive complexes play a role in FOXP3 target gene regulation, but deeper mechanistic insight is incomplete. We have now specifically identified the polycomb-repressive complex 1 (PRC1) family member, BMI1 in the regulation of a proinflammatory enhancer network in both human and murine Tregs. Using human Tregs and lamina propria T cells, we inferred PRC1 to regulate Crohn's associated gene networks through assays of chromatin accessibility. Conditional deletion of BMI1 in murine FOXP3+ cells led to systemic inflammation. BMI1-deficient Tregs beared a TH1/TH17-like phenotype as assessed by assays of genome wide transcription, chromatin accessibility and proteomic techniques. Finally, BMI1 mutant FOXP3+ cells did not suppress colitis in the adoptive transfer model of human inflammatory bowel disease. We propose that BMI1 plays an important role in enforcing Treg identity in vitro and in vivo. Loss of Treg identity via genetic or transient BMI1 depletion perturbs the epigenome and converts Tregs into Th1/Th17-like proinflammatory cells, a transition relevant to human Crohn's disease associated CD4+ $\mathrm{T}$ cells.
\end{abstract}

\section{Introduction}

Persistent activation of pathogenic adaptive immune cells is evident in many autoimmune diseases, including inflammatory bowel disease (IBD, including Crohn's disease, CD, and ulcerative colitis [UC], refs. 1-3). In addition, incapacitation of the regulatory arm of the adaptive immune system can perpetuate disease severity (4-6). Despite recent advances in the discovery of molecular and cellular mechanisms driving effector $\mathrm{CD} 4^{+} \mathrm{T}$ cell pathophysiology within IBD $(7,8)$, the potential role of Treg dysfunction is poorly understood. Furthermore, the dysregulated epigenetic events that lead to Treg dysfunction are not well defined. Thus, there is a need to fill this gap in knowledge to better understand and manage these diseases.

Among the adaptive immune cells, the $\mathrm{CD} 4^{+} \mathrm{CD} 25^{\text {hi }}$ Tregs, which are marked by the expression of the X-chromosomeencoded forkhead domain protein 3 (FOXP3), are critical for maintaining immune self-tolerance and suppressing inflammatory responses $(9,10)$. In fact, Treg development and maintenance is dependent on FOXP3 (11-13). Consequently, loss of function of this lineage-defining transcription factor by deletion or genetic mutation leads to autoimmunity, as is evident in human immune dysregulation, polyendocrinopathy, enteropathy, X-linked (IPEX)

Conflict of interest: The authors have declared that no conflict of interest exists. Copyright: () 2021, American Society for Clinical Investigation.

Submitted: June 4, 2020; Accepted: April 15, 2021; Published: June 15, 2021.

Reference information: J Clin Invest. 2021;131(12):e140755.

https://doi.org/10.1172/JCl140755. syndrome and multiorgan inflammation in mice $(4,14,15)$. This clear association to disease emphasizes the importance of FOXP3-mediated Treg dysfunction to the pathobiology of several debilitating inflammatory diseases.

FOXP3-mediated Treg development and immunosuppressive function relies on chromatin re-organization (16). This epigenetic control of gene expression is relevant, not only to Treg function but also to a plethora of immune cells and thereby underpins the pathogenesis of many human diseases $(17,18)$. Elegant work has shown that epigenomic remodeling, and in particular, nucleosome positioning and posttranslational histone modifications (histone marks), regulates gene transcription, driving T cell differentiation, stability, and function (19-23). These modifications are predominantly orchestrated by transcription factors which in turn recruit various nuclear macromolecules. This results in active regions becoming highly accessible while repressed regions are less accessible. Thus, chromatin accessibility, defined as the degree to which nuclear macromolecules are able to physically interact with chromatinized DNA, is determined by the occupancy and topological organization of appropriately marked nucleosomes.

Several laboratories, including ours, have shown that histone modifying enzymes, and in particular, the enhancer of Zeste homo$\log 2$ (EZH2), play a critical Treg-specific role in preventing multiorgan inflammation and colitis (24-26). EZH2, the enzymatic subunit of the Polycomb repressive complex 2 (PRC2), catalyzes the tri-methylation of lysine 27 (K27) on histone 3 (H3K27me3; refs. 27, 28). In the canonical model of Polycomb-mediated gene repression, the Polycomb repressive complex 1 (PRC1), through its chromobox (CBX) 
A

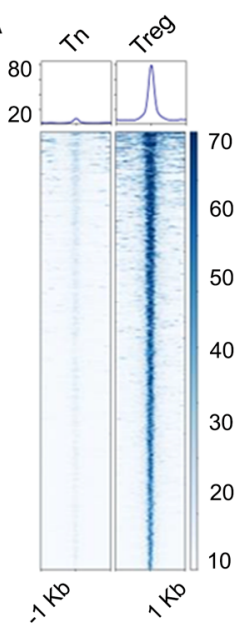

ATAC peaks
B
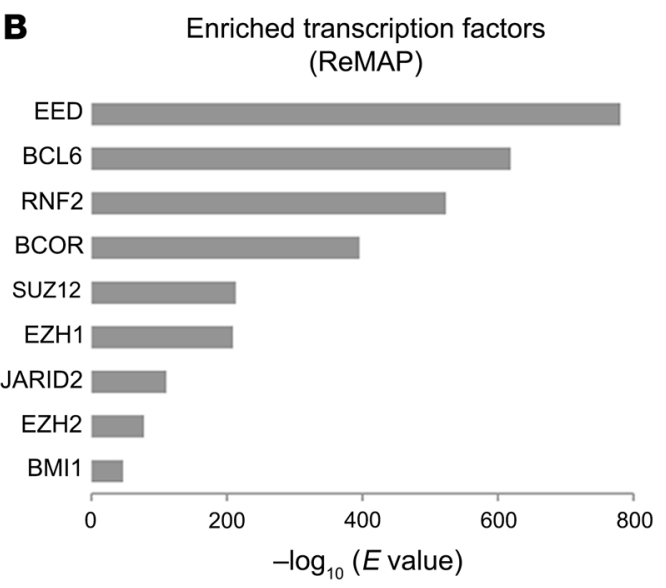

C

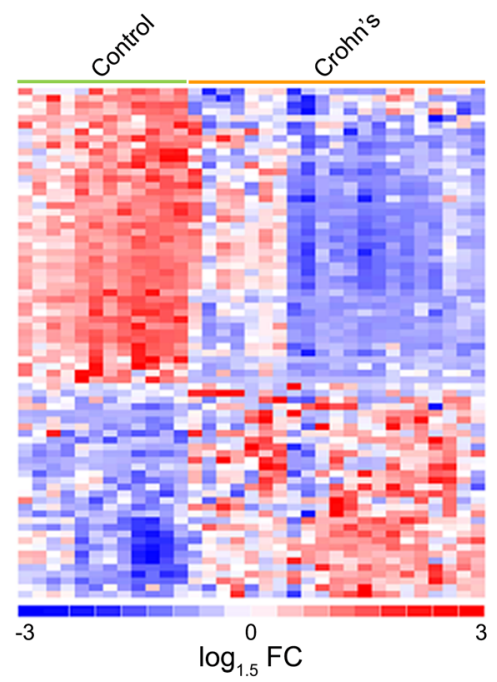

D

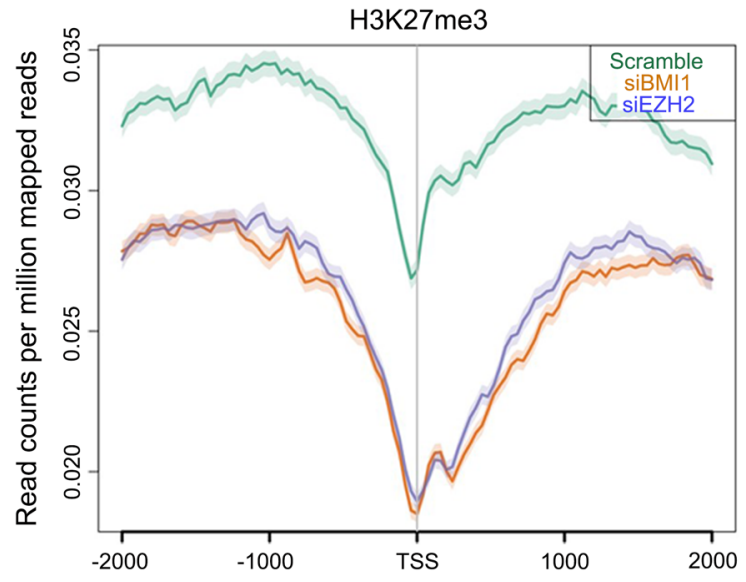

E

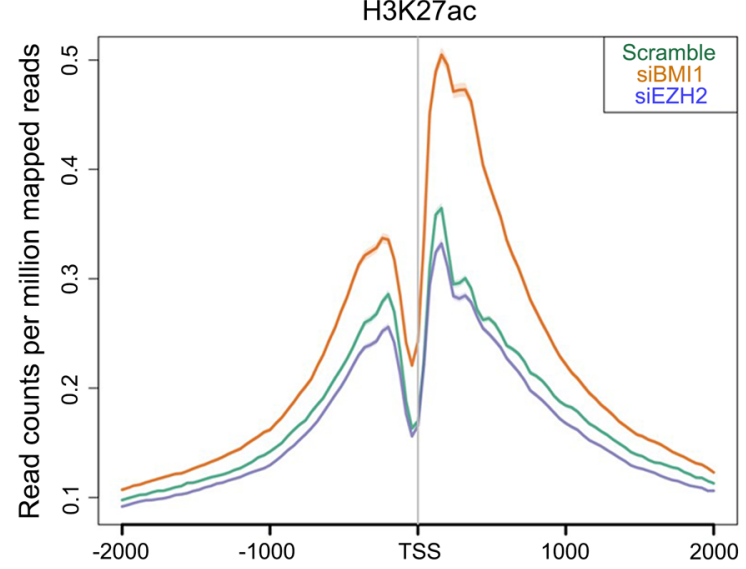

Figure 1. Members of PRC1 and PRC2 complexes are highly enriched at ATAC peaks associated with Crohn's disease-related genes. (A) Average binding profiles representing open chromatin state at ATAC peaks associated with Crohn's disease-related genes in in vitro-generated Tregs derived from PBMCs. ATAC regions are shown to be significantly more open in Treg cells compared with T-naive cells at day 7. (B) Enrichment scores of members of PRC1 and PRC2 at ATAC peaks associated with disease-related genes from ENCODE ChIP-Seq data sets calculated using the ReMAP tool. (C) Heat map depicting PRC1 differentially regulated genes in T cells from Crohn's patients compared with healthy individuals (>1.5 fold change, 76 genes) assessed by RNA sequencing from $\mathrm{CD} 4^{+} \mathrm{T}$ cells isolated from terminal ileum resections and enriched within known PRC1 targets. Upregulation is presented in red and downregulation in blue with intensity correlating to log fold change. (D) Chromatin immunoprecipitation with genetic knockdown of BMI1 (red) or EZH2 (blue) in Jurkat cells showing global disruption of H3K27me3 at the TSS compared with scramble (green). (E) Increase in H3K27ac marks in the absence of BMI1 (red) compared with EZH2 (blue) and scramble (green).

domain proteins, recognizes the PRC2-established H3K27me3 mark, allowing it to physically interact with chromatin (29). In this manner, PRC1 is known to maintain the repressed state of genes marked by PRC2. Interestingly, multiple PRC1 subcomplexes have been described, each with distinct biochemical properties. However, the common enzymatic subunit of all these complexes is the obligate heterodimer of RING1A/B and 1 of 6 PCGF homologs. RING1A/B catalyzes ubiquitination of lysine 119 (K119) on histone 2A (H2A; H2AK119ub; ref. 30). Indeed, 2 PCGF homologs, BMI1 and MEL18, support the enzymatic function of RING proteins, and their dysfunction has been implicated in human disease pathogenesis (31). These stepwise events induced by PRC 2 and PRC1 initiate, propagate, and maintain target gene repression in an evolutionarily conserved manner (32). Despite this accepted importance of PRC2 in Treg biology, the field has been limited to murine studies of conditional deletion of the histone methyltransferase EZH2 in the FOXP3 (or CD4)- expressing T cell $(16,20,25,33)$. Given the emerging role for Polycomb complexes in immune regulation broadly and the emergence of Polycomb targeted therapies in the clinic, it is important to investigate more nuanced Polycomb-mediated regulatory events, particularly in an inflammatory disease context (i.e., human CD). Here, we here report the crucial role for PRC1 and in particular, BMI1, in Treg function. We further explore the loss of PRC1-mediated gene networks as a hallmark of CD-associated lymphocytes. This not only advances our mechanistic knowledge on the role of polycomb-mediated events in inflammatory diseases, but also contributes to the design of clinical studies that aim at targeting this protein for therapeutic purposes, both of which have significant biomedical relevance.

\section{Results}

The PRC1 is implicated in the regulation of CD-associated gene networks. Our previously published work demonstrated deregulation 

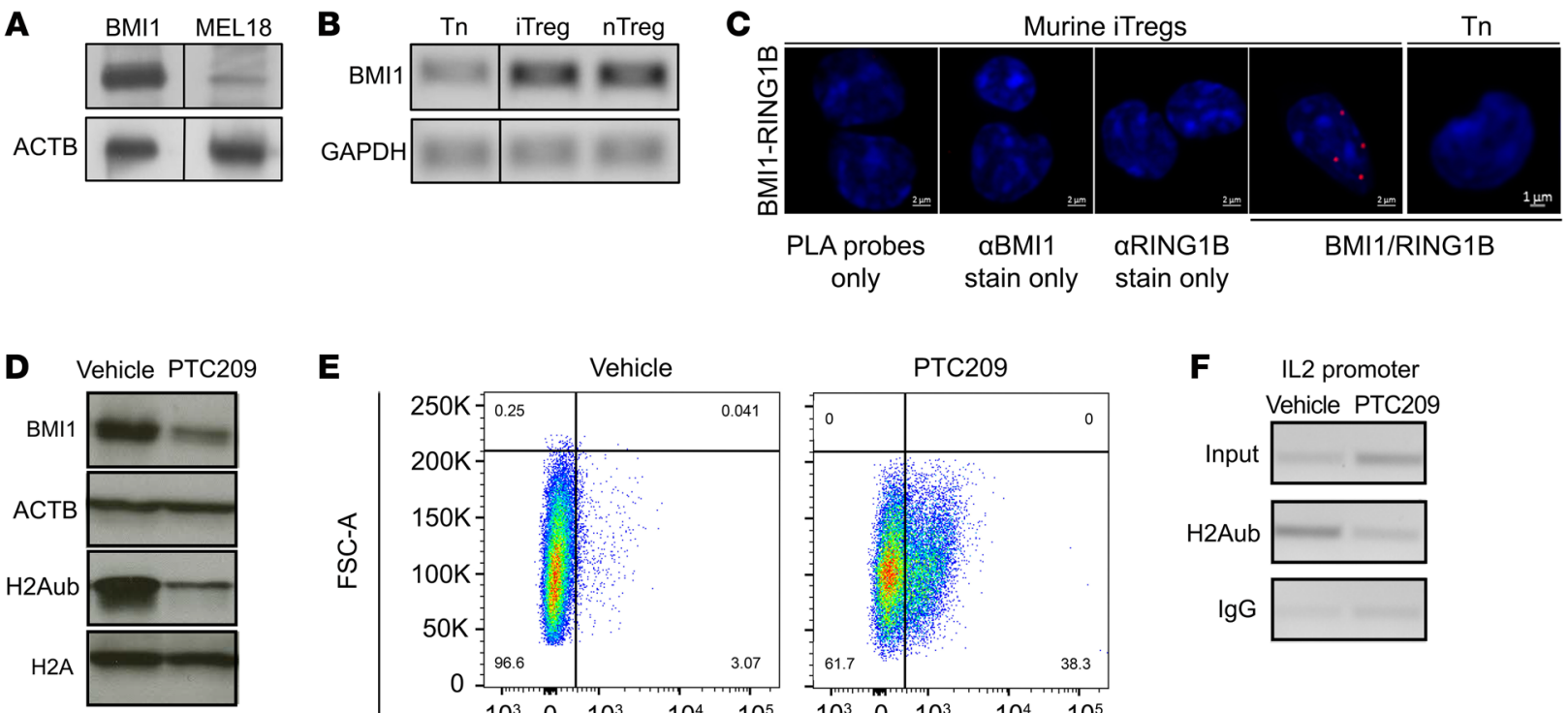

$\mathbf{E}$

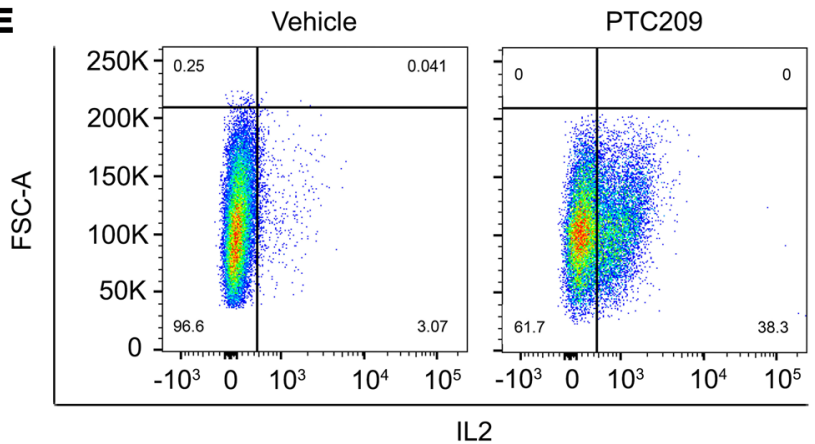

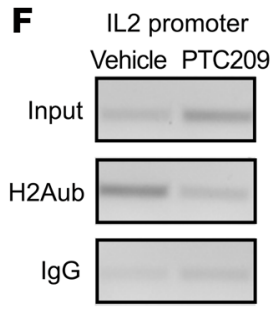

Figure 2. The canonical PRC1 member BMI1 is uniquely expressed in murine lymphocytes. (A) Representative IB analysis of BMI1 and MEL18 protein expression in freshly isolated murine CD25+⿱ Tregs. (B) Upregulation of BMI1 mRNA upon in vitro induction of murine Tregs from naive cells cultured under polarizing conditions with TGF- $\beta 1$ and IL-2 for 5 days. (C) Representative PLA images demonstrate the interaction between BMI1 and RING1B (red dots) present in murine iTregs vs naive CD4 ${ }^{+}$T cells (7.8 \pm 6.3 vs. $0.35 \pm 0.45, P=0.03, n=4$ mice per group). The units for PLA are an average of the number of dots (red dots) present within cells, representing BMI1-RING1B protein complexes. (D) Representative IB analysis depicts decreased protein expression of BMI1 and H2AK119ub expression in human T cell leukemic line (Jurkat E6 cells) upon pharmacologic treatment with the BMI1 inhibitor, PTC209. (E) IL2 expression in Jurkat cells upon pharmacologic inhibition of BMI1 with PTC209 measured by fluorescent activated cell sorting (FACS) and flow cytometric analysis. (F) Chromatin immunoprecipitation and quantitative PCR depicts a global loss of H2AK119 ubiquitination mark on the IL2 promoter in Jurkat cells in the presence of the BMI1 inhibitor, PTC209, respectively.

of FOXP3-mediated gene networks in CD4+ lymphocytes isolated from ileal active $\mathrm{CD}$ (25). To understand the epigenetic drivers of FOXP3 de-repression in human CD, we integrated the chromatin accessibility landscape of human Tregs using ATAC-Seq (assay for transposase-accessible chromatin using sequencing) with differentially expressed genes (DEGs) in $\mathrm{CD}$-associated $\mathrm{CD} 4^{+}$ lymphocytes (CD-DEGs). The heatmap in Figure 1A demonstrates curation of the full chromatin accessibility heatmap (Supplemental Figure 2; supplemental material available online with this article; https://doi.org/10.1172/JCI140755DS1) for CD-DEGs (Supplemental Table 1). To infer mechanistic information related to DNA/chromatin binding proteins regulating this $\mathrm{CD}$ relevant gene program, these areas of enhanced chromatin accessibility were cross-referenced with public and ENCODE ChIP-Seq data sets using ReMap 2018 v1.2 (Figure 1B and Supplemental Table 2). Congruent with the critical role for PRC2 in Tregs $(16,25)$ and validating our methodological approach, we observed a significant association of accessible chromatin areas with the PRC2 obligate family members, embryonic ectoderm development (EED), Suppressor of zeste 12 (Suz12), and EZH2 (Figure 1B). In addition, we identified areas of open chromatin at loci encoding both canonical and noncanonical PRC1 family members (RNF2, BCOR, and BMI1), revealing for the first time a function for these epigenomic regulators in the Treg developmental process.

To shed further light into the association of this complex with human CD, we quantified the enrichment of known PRC1 gene targets (34) within CD-DEGs. We identified that $41 \%$ of published PRC1 target genes were indeed enriched ( 76 of 186 genes, $\chi^{2}$ test
$P=1.078 \times 10^{-6}$ ) within the 1279 upregulated CD-DEGs (Figure 1C). Moreover, we find that chemokine and cytokine signaling, among other inflammatory pathways, are the inferred phylogeny and function of this list of PRC1 target genes (Panther 2016, $P=$ 0.006; Supplemental Figure 1A). Taken together, these investigations seem to support a role for PRC1 in human CD, and justify deeper mechanistic studies.

To begin to appreciate a PRC2-dependent or independent role for PRC1 in human lymphocytes, we used RNAi-mediated depletion of BMI1 (core activity of PRC1) or EZH2 (core activity of PRC2) expression in the experimentally well-characterized human Jurkat leukemia cell model (Supplemental Figure 1B). Next, we performed genome-wide chromatin immunoprecipitationsequencing (ChIP-Seq) analyses for H3K27me3 and H3K27ac in chromatin isolated from BMI1-depleted or EZH2-depleted cells. The aggregate plot from scramble-transfected control cells (Figure 1D, green line) displays genomic regions near transcriptional start sites (TSS) that were marked by H3K27me3. Consistent with the established biology of PRC1 in maintaining PRC2-deposited H3K27me3 marks, depletion of BMI1 (Figure 1D, red line) or EZH2 (Figure 1D, blue line) abrogated the global deposition of H3K27me3 compared with control cells. Surprisingly, we observed a robust enhancement of the H3K27ac marks across both TSS and intragenic regions only in BMI1-deficient cells (Figure 1E). These data suggest that, although PRC2 and PRC1 cooperatively enforce the H3K27me3 repressive mark across the genome, there exists a PRC2-independent function for PRC1 in the remodeling of the human lymphocyte epigenome. 
A

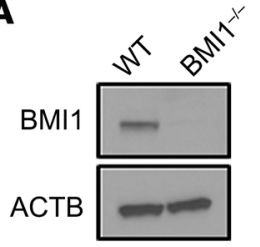

B

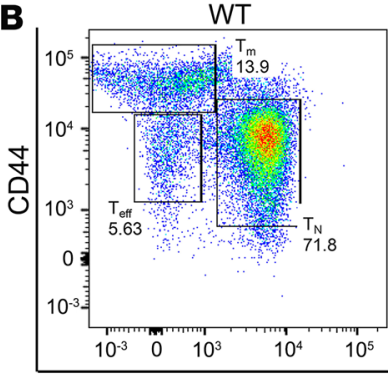

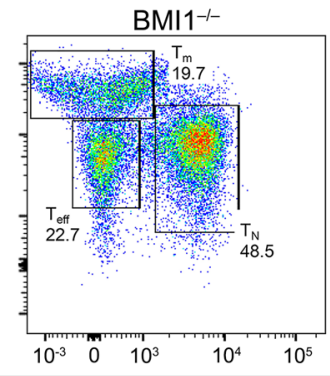

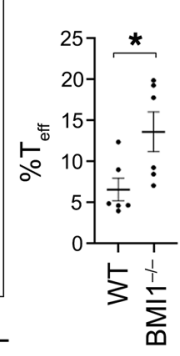

C

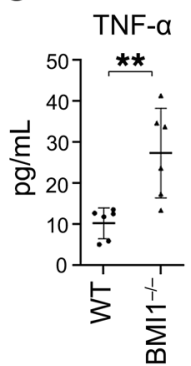

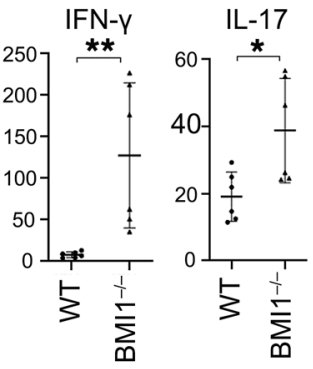

D

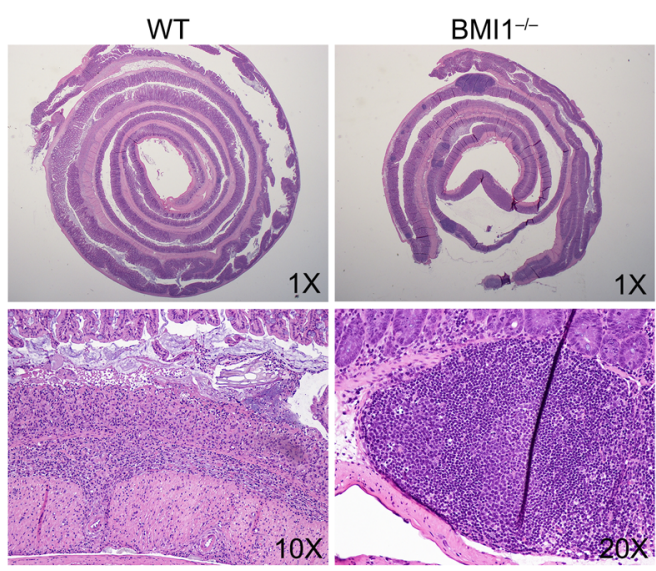

Figure 3. Characterization of conditional BMI1-deficient mice reveals a proinflammatory phenotype. (A) Representative IB analysis for BMI1 protein expression in Tregs

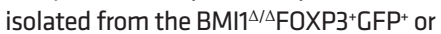
WT mouse. (B) Flow cytometric analysis of peripheral $C D 4^{+} T$ cells showing a higher percentage of effector (CD62 ${ }^{10}$ CD44int) and memory (CD62 $\left.{ }^{\text {lo }} \mathrm{CD} 44^{\text {hi }}\right) \mathrm{T}$ cells relative to naive (CD62 $\mathrm{L}^{\text {hi) }}$ undifferentiated T cells $\left(13.64 \% \pm 2.429 \%\right.$ of total $C D 4^{+} T$ cells vs. $6.618 \% \pm 1.373 \%, P=0.03$ ). (C) Cytokine analysis from peripheral blood shows an increased concentration of TNF- $\alpha$ ( $27.4 \mathrm{pg} / \mathrm{dL} \pm 10.9$ vs. $10.3 \pm 3.8 \mathrm{pg} / \mathrm{dL}, P=0.004)$, IFN $-\gamma(127.6 \mathrm{pg} /$ $\mathrm{dL} \pm 87.2$ vs. $8.0 \pm 3.6 \mathrm{pg} / \mathrm{dL}, P=0.002)$, and IL-17 (38.9 pg/dL \pm 15.6 vs. $19.2 \pm 7.3 \mathrm{pg} / \mathrm{dL}$, $P=0.04)$ in conditional BMI-deficient mice. (D) Histopathology from colonic samples shows increased number and size of lymphoid aggregates of the conditional BMI1-deficient mice. Data for serum cytokine analysis are from 6 biological replicates (unique animals). Error bars denote SD. Nonparametric unpaired $t$ test was performed using Mann-Whitney $U$ test, and $P<0.05$ was considered statistically significant. ${ }^{*} P<0.05$ and ${ }^{* *} P<0.01$
Canonical PRC1 members are expressed, heterodimerize, and have regulatory function in Treg cells. To further deduce the immunologic relevance of the PRC1 complex in primary cells we utilized in vitro and in vivo systems of BMI1 deficiency in primary FOXP3-expressing Treg cells. To derive the proper system in which to study PRC1 function in subsequent in vivo experiments, we investigated whether Tregs express 2 key PRC1 family members, MEL18 (PCGF2) and BMI1 (PCGF4). BMI1 and MEL18 are orthologs and mutually exclusive PCGF members of the canonical PRC1 complex that are required for the ability of PRC1 to monoubiquitinate histone $\mathrm{H} 2 \mathrm{~A}$ $(35,36)$. Thus, we performed Western blot analysis of freshly isolated murine splenic Tregs with specific antibodies against BMI1 and MEL18. This experiment showed abundant and constitutive expression of BMI1 in murine Tregs (Figure 2A). Congruently, both freshly isolated natural Tregs and induced Tregs also display an increased expression of BMI1 mRNA when compared with naive $\mathrm{CD}^{+} \mathrm{T}$ cells (Figure 2B). As an obligate heterodimer to the enzymatically active RING1B protein, BMI1 is requisite to the functional activity of PRC1 (30). Thus, given the inference of BMI regulation of human epigenetic remodeling in Tregs (Figure 1B), we visually and quantitatively probed for BMI1-RING1B protein interaction through in situ proximity ligation assay (PLA) with antibodies specific for both of these proteins with subsequent confocal imaging of murine cells. We found that, in agreement with our ATAC-Seq data, Tregs uniquely displayed RING1B-BMI1 protein complexes in contrast to naive cells (7.8 \pm 6.3 vs. $0.35 \pm 0.45, P=0.03$; Figure $2 \mathrm{C}$ ). The units for PLA are an average of the number of dots (red dots) present within cells, representing BMI1-RING1B protein complexes. These data conclusively show the presence of BMI1 as well as its heterodimerization with RING1B specifically in Treg cells, suggesting functional relevance of PRC1 in Treg differentiation and function.
PRC1 maintains the repressed configuration of PRC2marked chromatin through its ability to serve as the writer of the H2AK119ub mark. Given that interleukin 2 (IL-2) is an established target of PRC2 (24), we hypothesized that increased IL-2 cytokine production may serve as a functional readout to test BMI1 repressive function in lymphocytes. Using Western blot studies, we first confirmed that the treatment of Tregs with the specific pharmacologic transcriptional inhibitor of BMI1, PTC209 (37), led to the global decrease of BMI1 and the H2AK119ub mark in Jurkat cells but not in vehicle-treated controls (Figure 2D). Moreover, we measured IL-2 expression in PTC209-treated cells via flow cytometric analysis as well as 2 control genes (CCL2 and ATP2B4; Supplemental Figure 3) known to be regulated by BMI1 and associated with $\mathrm{H} 3 \mathrm{~K} 27 \mathrm{ac}$ peaks in BMI1-deficient Jurkat cells. The results of these experiments show that BMI1 inhibition leads to an increase in IL-2 production and the concomitant decrease in the H2AK119ub mark at the IL2 gene promoter (Figure 2, E and F). Together, these data demonstrate that in Tregs, the canonical PRC1 family members are not only expressed and physically interact but also modulate cytokine expression, as evidenced by IL-2 production.

Conditional deletion of BMI1 specifically in FOXP3 cells leads to a pro-inflammatory murine phenotype. To investigate the immunologic relevance of the PRC1 complex in primary cells, we next created and utilized an in vivo system of FOXP3-driven BMI1 deficiency. To achieve this, we crossed Foxp3-Cre [B6- $\mathrm{Tg}$ (Foxp $\left.3^{\text {EGFP-Cre })} 1 \mathrm{cJbs} / \mathrm{J}\right]$ and Bmi1-flox C57/BL6 animals to efficiently inactivate BMI1 in FOXP3-expressing $\mathrm{CD}^{+}{ }^{+} \mathrm{T}$ cells $\left[\mathrm{BMI}^{-/-} \mathrm{FOXP}^{+} \mathrm{GFP}^{+}\right]$(Figure $3 \mathrm{~A})$. We observed that both the homozygous BMI1-deficient littermates and heterozygous $\mathrm{BMI}^{-/+}$animals were, like their WT littermates, born in the usual Mendelian ratios without obvious gross phenotypic abnormalities. However, immunologic assessments of 
A

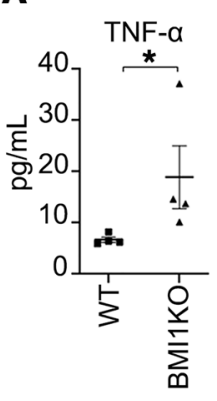

CD25 $5^{\text {hi }}$ supernatants

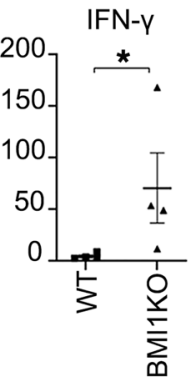

IL-17

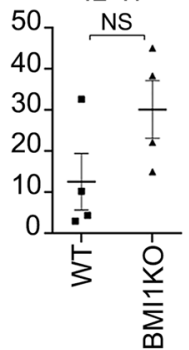

B

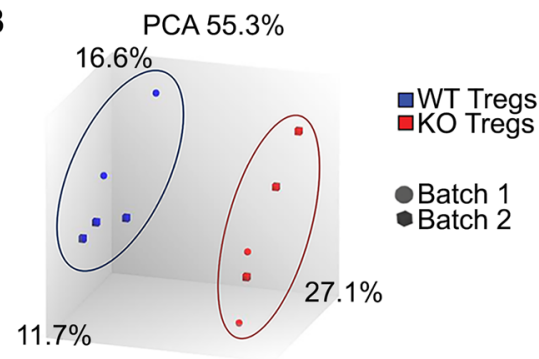

C

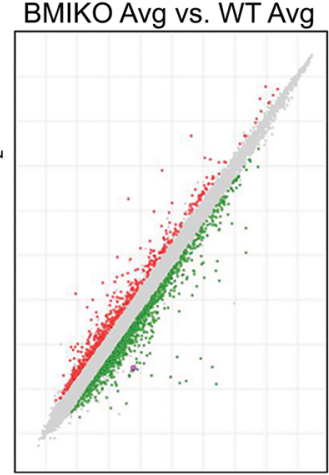

WT Avg $\left(\log _{2}\right)$
D

Positive regulation of TNF signaling

Regulation of TNF signaling

Regulation of NFKB transcription factor activity

Regulation of microtubule nucleation

Positive regulation of cytokine signaling

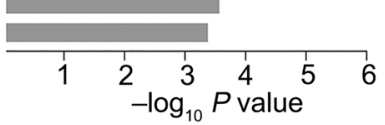

$\mathbf{E}$

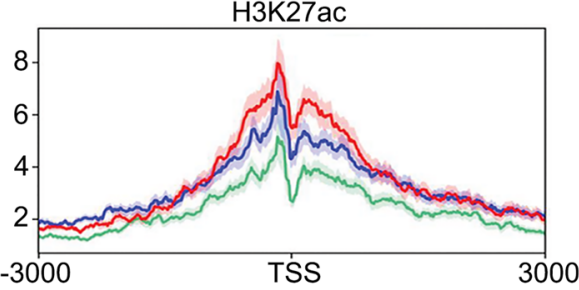

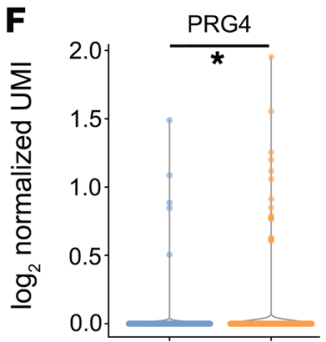
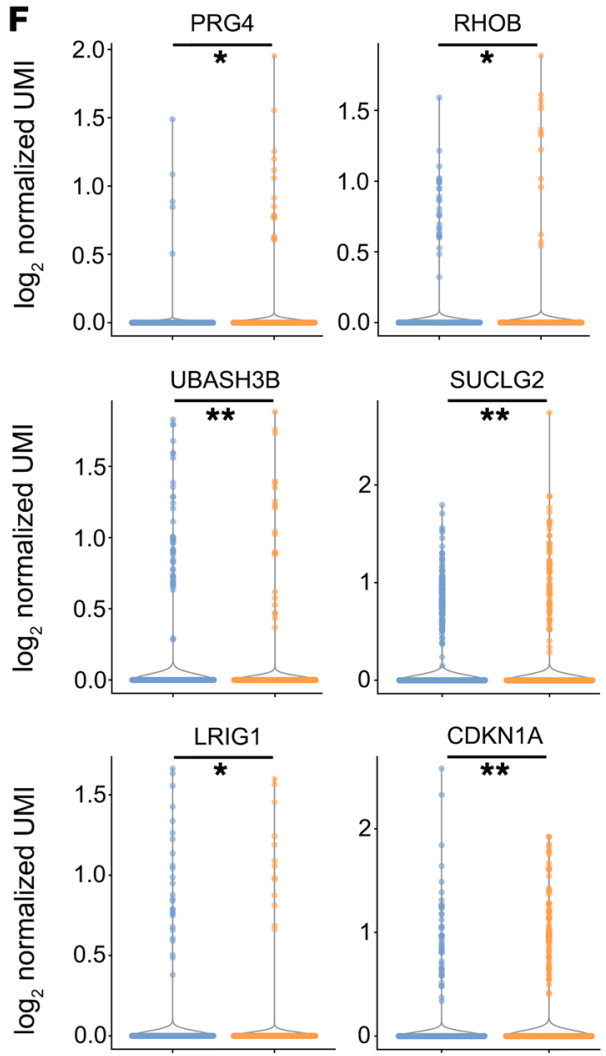
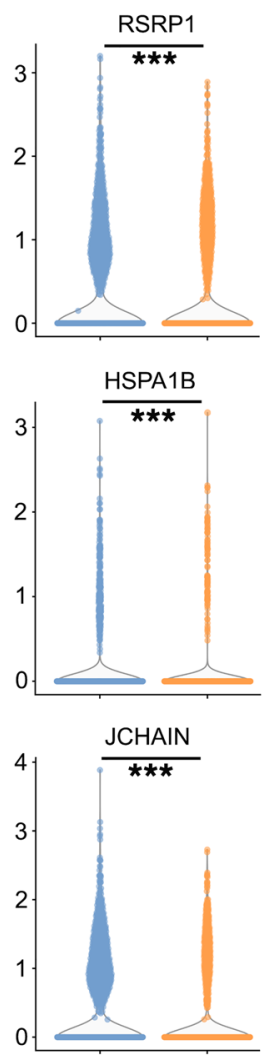
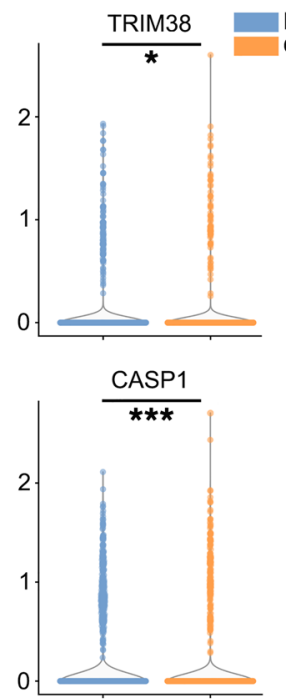

PPP1R3E

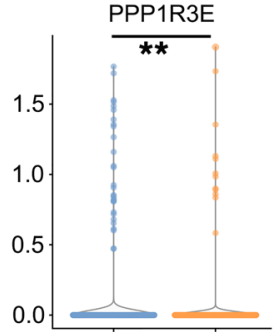

Figure 4. BMI1 knockdown specifically upregulates H3K27ac occupancy at TSS of regulated genes and is relevant to human CD. (A) Splenic Tregs isolated from conditional BMI1 KO mice showed expression of proinflammatory cytokines TNF- $\alpha$ (18.9 pg/dL \pm 12.3 vs. $6.7 \pm$ $1.0 \mathrm{pg} / \mathrm{dL}, P=0.029)$ and IFN- $\gamma(70.9 \pm 67.6 \mathrm{pg} /$ dL vs. $4.9 \pm 2.9 \mathrm{pg} / \mathrm{dL}, P=0.029$ ) by multiplex cytokine analysis. (B) Principal component analysis separates $\mathrm{BMI1}^{-1-} \mathrm{FOXP3}^{+}$cells (red) from WT (blue) Tregs (data representative of 5 mice/condition in 2 separate experiments). (C) Upregulated (red) and downregulated (green) differentially expressed genes that separate murine BMI1 ${ }^{-1-} \mathrm{FOXP3}^{+}$cells from WT Tregs. (D) Gene ontology analysis (GO Biological Process 2018b) of the 130 upregulated genes identified in resting $\mathrm{BMI1}^{-1-} \mathrm{FOXP3}^{+}$compared with WT Tregs (Enrichr, Ma'ayan Lab). (E) Average occupancy profiles of H3K27ac in Jurkat cells at TSS of human homologs of genes upregulated in BMIKO mice. (F) Violin plots demonstrating enrichment of 12 BMI1KO genes within the Treg-specific scRNAseq data derived from patients with Crohn's disease displaying a CIMATS phenotype. The data for the cytokines are generated from $n=4$ animals, in triplicates Nonparametric unpaired $t$ test was performed using Mann-Whitney $U$ test, and $P<0.05$ was considered statistically significant. Numerical values reflecting means \pm standard deviation. Means obtained from average in triplicates from each mouse. Error bars denote SEM. 

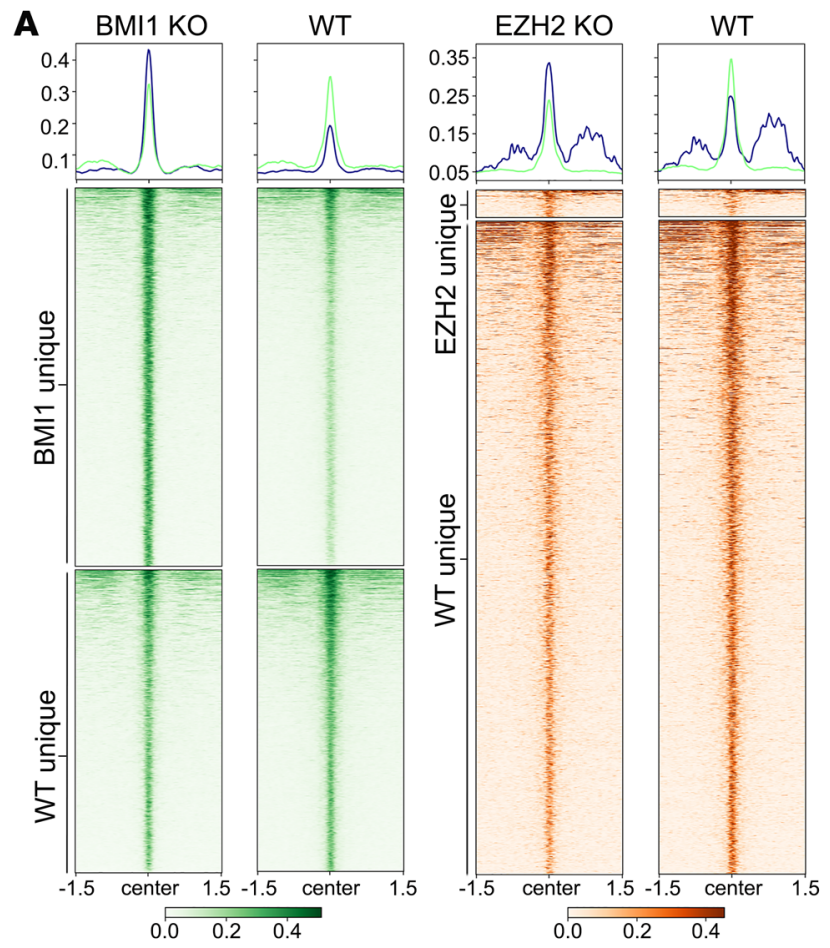

6-week old $\mathrm{Bmi1}^{-/-} \mathrm{Foxp}^{+} \mathrm{GFP}^{+}$animals using flow cytometry and in vitro assays of cellular response demonstrated an expansion of an effector/memory $\mathrm{CD}^{+} \mathrm{T}$ cell population relative to control animals $\left(13.64 \% \pm 2.429 \%\right.$ of total CD $4^{+} \mathrm{T}$ cells vs $6.618 \% \pm 1.373 \%$, $P=0.03$, Figure $3 \mathrm{~B})$. In addition, multiplex analysis of serum cytokines from $\mathrm{BMI1}^{-/-} \mathrm{FOXP3}^{+} \mathrm{GFP}^{+}$mice via cytometric bead array indicated an activated immune response, as shown through the significantly elevated concentration of TNF- $\alpha(27.4 \mathrm{pg} / \mathrm{dL} \pm 10.9$ vs. $10.3 \pm 3.8 \mathrm{pg} / \mathrm{dL}, P=0.004)$, IFN- $\gamma(127.6 \mathrm{pg} / \mathrm{dL} \pm 87.2$ vs. $8.0 \pm$ $3.6 \mathrm{pg} / \mathrm{dL}, P=0.002)$, and IL-17 (38.9 pg/dL \pm 15.6 vs. $19.2 \pm 7.3 \mathrm{pg} /$ $\mathrm{dL}, P=0.04$ ), compared with control animals (Figure $3 \mathrm{C}$ ). Moreover, while gross anatomical assessment of organs in 6 week-old animals did not reveal end organ pathology, $\mathrm{Bmi1}^{--} \mathrm{Foxp}^{+} \mathrm{GFP}^{+}$ animals aged by 19 to 21 weeks exhibited a mild neutrophilic infiltrate and an increase in number and size of lymphoid aggregates limited to the large intestine (Figure 3D). Thus, this data derived from our genetically-engineered mouse model is congruent with both our chromatin accessibility and in vitro functional assays and clearly demonstrates that the Treg-specific genetic ablation of BMI1 in vivo induced a spontaneous inflammatory phenotype with concomitant elevation of Th1/Th17-associated proinflammatory cytokines. These findings suggest a role for this pathway in maintaining the proper immune homeostasis of the colon.

BMI1-deficient FOXP3 $3^{+}$cells display a Crohn's associated proinflammatory phenotype. We examined the cell differentiation and function of Tregs ex vivo by isolating these cells from $\mathrm{Bmil}^{-/} \mathrm{Foxp}^{+} \mathrm{GFP}^{+}$ mice. Briefly, freshly isolated splenic Tregs $\left(\mathrm{CD} 4^{+} \mathrm{CD} 25^{++} \mathrm{FOXP} 3^{+}\right.$ $\mathrm{GFP}^{+}$) from WT and $\mathrm{Bmil}^{-/-}$animals were stimulated with $\alpha \mathrm{CD} 3 /$ CD28 for 24 hours. Supernatants collected from BMI1 ${ }^{-/-}$Tregs displayed significantly elevated expression of the proinflammatory effector cytokines TNF- $\alpha(18.9 \mathrm{pg} / \mathrm{dL} \pm 12.3$ vs. $6.7 \pm 1.0 \mathrm{pg} / \mathrm{dL}, P=$ $0.029)$ and IFN- $\gamma(70.9 \pm 67.6 \mathrm{pg} / \mathrm{dL}$ vs. $4.9 \pm 2.9 \mathrm{pg} / \mathrm{dL}, P=0.029)$ in

\begin{tabular}{|c|c|c|}
\hline Sequence & Motif & $P$ value \\
\hline & BORIS & $1 e-1127$ \\
\hline AACAGGAAGT & Ets1 & $1 e-609$ \\
\hline GIGAAAGT & IRF1 & $1 e-198$ \\
\hline TGTGGITACA & RUNX1 & 1e-197 \\
\hline ATCCCCA & NFkB-p65 & $10-$ \\
\hline
\end{tabular}

Figure 5. BMI1-deficient Tregs show unique open regions with unique transcriptional regulatory nodes. (A) Differentially open chromatin peaks in BMI1-deficient (BMI1 KO) and EZH2-deficient (EZH2 KO) murine splenic Tregs compared with WT Tregs. (B) Top 5 DNA-binding transcription factors identified by HOMER de novo motif analysis on the 5181 open chromatin regions unique to $\mathrm{BMI1}^{-1-}$ Tregs.

contrast to WT Tregs (Figure 4A). Hence, this intrinsic effector-like phenotype of activated $\mathrm{BMI}^{-/-}$Tregs implies a BMI1-dependent inhibition of genes encoding these proinflammatory cytokines.

To further extend these compelling results, we performed unbiased microarray analysis of freshly sorted $\mathrm{GFP}^{+}$cells. Unsupervised principal component analysis (PCA) of the results shows that both resting WT and $\mathrm{BMI}^{-/-}$Tregs cluster into 2 distinct groups reflecting their overall differences in gene expression patterns (Figure 4B). Differential gene expression analysis between WT and BMI1 ${ }^{-/-}$Tregs revealed 421 DEGs. Additional analyses show that out of these 421 DEGs, 130 (30.88\%) and 291 (69.12\%) genes were upregulated and downregulated, respectively (Figure 4C). Furthermore, ontological analysis of the upregulated genes found in $\mathrm{BMI}^{-/-}$Tregs demonstrate an enrichment of TNF- $\alpha$ and NF- $\kappa \mathrm{B}$ biological processes (Figure 4D). Congruent with the transient BMI1 knockdown experiments in human cells, these upregulated genes found in BMI1-deficient Tregs were also found to be marked by H3K27ac (Figure 4E).

To heighten the relevance to human $\mathrm{CD}$, we examined the expression of 62 confident DEGs (>2-fold change differential expression upon BMI1 KO) within a single cell sequencing data set derived from CD associated ileum (38). Remarkably, 12 of 62 genes were found to be differentially expressed within Treg cells comprising a module associated with refractory CD (GIMATS module, Figure $4 \mathrm{~F}$ ). The association of these 12 genes was significantly seen only among the Treg population and not in other T cell subsets, such as cytotoxic T cells (Supplemental Figure 5). These data suggest that as evidenced in mice, BMI1 plays a role in repression of Treg specific inflammatory pathways relevant to the inflamed mucosa of CD.

A PRC2-independent role for PRC1 in Treg cells is evident. PRC2 and PRC1 functional cooperation is known to exist in many cell types; thus using genetic KO mouse lines we directly tested for PRC2-independent function of PRC1. We performed ATAC- 
A

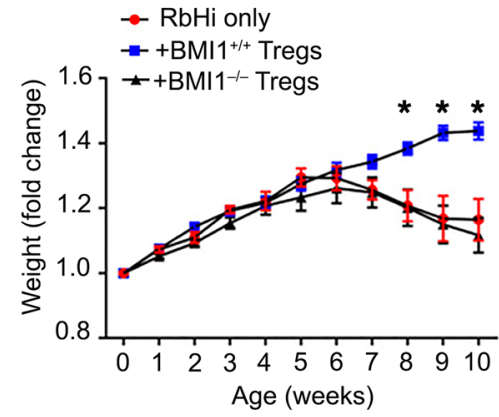

B

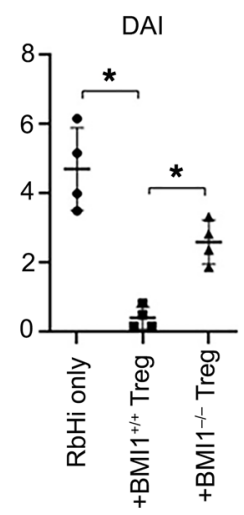

C

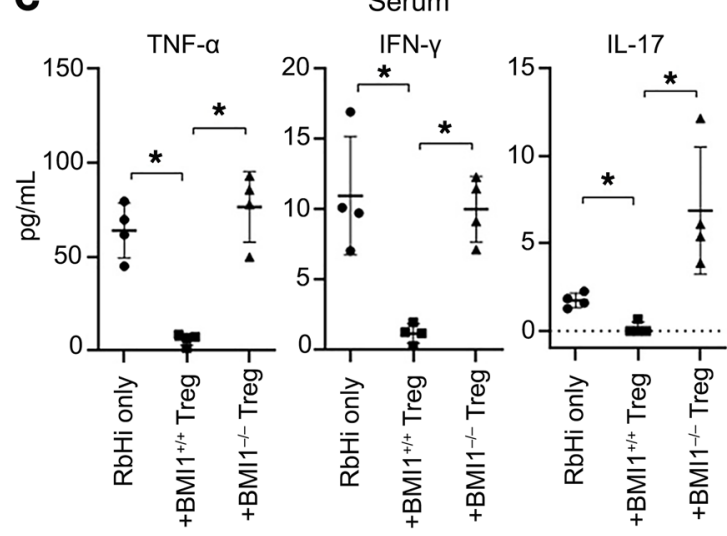

D
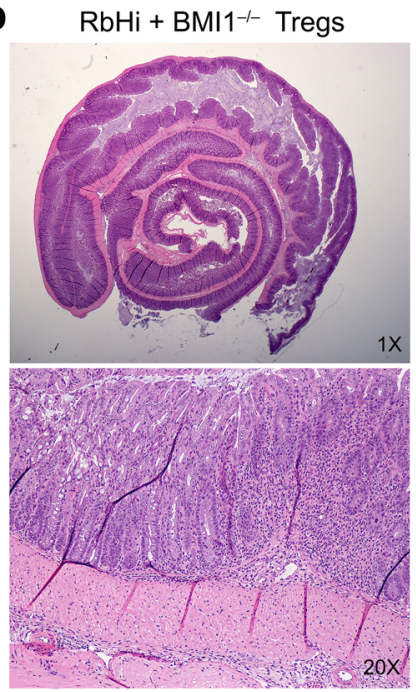

$\mathrm{RbHi}+\mathrm{BMI1}^{+/+}$Tregs

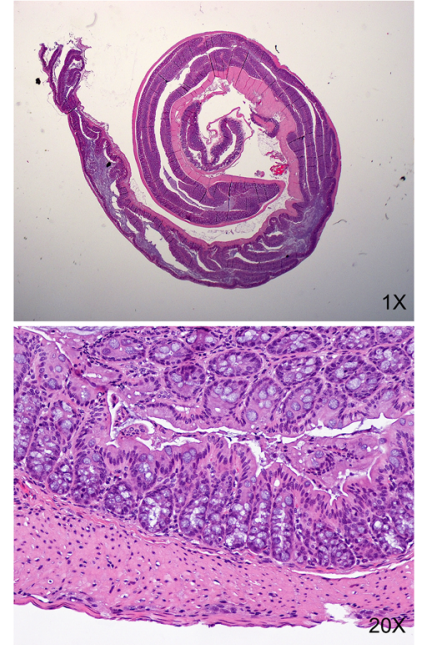

E Histologic score

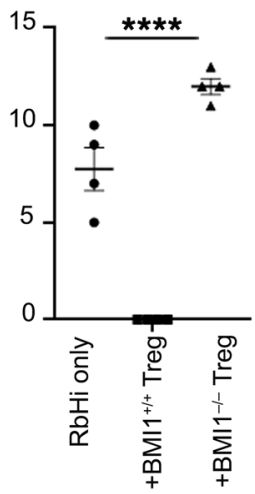

Figure 6. BMI1-deficient Tregs fail to suppress colitis in vivo. Mice subjected to T cell transfer model of colitis with concomitant injection of naive T cells with either BMI1/-- Tregs or WT Tregs. Mice injected with BMI1/- Tregs had (A) stunted weight gain (1.1 \pm 0.11 fold change from initial weight vs. $1.4 \pm 0.05$ fold change, $P=0.005)$ and (B) increased DAI when compared with mice injected with WT Tregs ( $2.6 \pm 0.63$ vs. $0.42 \pm 0.32, P=0.0048)$. The numerical values for DAI are the added product of a score calculated based appearance, stool consistency, and colon stiffness. (C) Cytokine analysis from peripheral blood showed an increased secretion of TNF- $\alpha(76.6 \pm 18.8 \mathrm{pg} / \mathrm{dL}$ vs. $6.0 \pm 3.2 \mathrm{pg} / \mathrm{dL}, P=0.028)$, IFN- $\gamma(10.0 \pm 2.3 \mathrm{pg} / \mathrm{dL}$ vs. $1.2 \pm 0.7 \mathrm{pg} / \mathrm{dL}, P=0.028)$, and IL-17 $(6.9 \pm 3.6 \mathrm{pg} / \mathrm{dL}$ vs. $0.2 \pm 0.3 \mathrm{pg} / \mathrm{dL}, P=0.028)$ in the mice receiving the BMI-deficient Tregs. (D) Histopathology of colon specimens revealed moderate colitis in mice injected with BMl1-deficient Tregs while mice receiving WT Tregs did not have evidence of colitis (histologic scores $12.0 \pm 0.82$ pg/dL vs. $0 \pm 0.00 \mathrm{pg} / \mathrm{dL}, P=0.029$ ). The data for the RbHi experiment are generated from $n=12$ animals, $4 /$ group. Nonparametric unpaired $t$ test was performed using Mann-Whitney $U$ test, and $P<0.05$ was considered statistically significant. For histologic score significance, Kruskal Wallis test was used for multiple comparisons, and $P<0.05$ was considered statistically significant. Numerical values reflecting means \pm standard deviation. Error bars denote SEM.

Seq on both freshly isolated BMI1-deficient and EZH2-deficient $\mathrm{FOXP}^{+}$murine lymphocytes. The results of this experiment, depicted as a heatmap in Figure 5A, shows the accessible chromatin regions found when comparing both EZH2- and BMI1deficient lymphocytes to WT Treg cells. Notably there are 5,181 BMI1-unique open regions compared with only 474 EZH2-unique open regions. Subsequent interrogation utilizing HOMER de novo motif discovery analyses on the unique 5,181 open chromatin regions in BMI1-deficient Tregs suggests that CTCF (analogous to Boris motif), Ets1, Runx1, and Irf family proteins (Figure 5B) may function as transcriptional regulatory nodes that are part of the response to BMI1 deletion in Tregs. Pathway analysis showed canonical immunologic inflammatory pathways (Supplemental Table 5). Similar analytical pathways for EZH2-unique open chromatin regions were less robust given the less frequent unique regions (474); yet are reported in Supplemental Table 6. Noteworthy, this set of transcription factors in Figure 5B were very recently described to regulate Th17 cellular development (39), which in light of our data suggest a novel link between BMI1 and Treg/Th17 lineage plasticity.

BMI1-deficient Treg cells fail to protect against colitis in vivo. Finally, we assessed the ability of BMI1-deficient Tregs to suppress intestinal inflammation in vivo utilizing an established experimental mouse model of $\mathrm{T}$ cell-induced colitis. We adoptively transferred $\mathrm{CD} 4^{+} \mathrm{CD} 45 \mathrm{Rb}^{\text {hi }} \mathrm{T}$ cells into RAG-1-deficient animals with cotransfer of splenic $\mathrm{WT}$ or $\mathrm{BMI1}^{-/-}$Tregs. We find that, in contrast to WT Tregs, the $\mathrm{BMI1}^{-/}$counterpart failed to suppress $\mathrm{T}$ cell-induced colitis in mice as reflected by stunted 
weight gain $(1.1 \pm 0.11$-fold change from initial weight vs. $1.4 \pm$ 0.05 -fold change, $P=0.005$, Figure 6A) and increased disease activity index (DAI) $(2.6 \pm 0.63$ vs. $0.42 \pm 0.32, P=0.0048$, Figure $6 \mathrm{~B}$ ). The numerical values for DAI are the added product of a score calculated based appearance (wasting 0-1, hunching 0-1), stool consistency (0-1), and colon stiffness (divided into proximal, medial, and distal; all $0-1$, then averaged). In agreement with our previous in vivo results, through the use of a multiplex cytokine assay, we observed elevated levels in serum TNF- $\alpha$ (76.6 $\pm 18.8 \mathrm{pg} / \mathrm{dL}$ vs. $6.0 \pm 3.2 \mathrm{pg} / \mathrm{dL}, P=0.028), \mathrm{IFN}-\gamma(10.0 \pm 2.3 \mathrm{pg} /$ $\mathrm{dL}$ vs. $1.2 \pm 0.7 \mathrm{pg} / \mathrm{dL}, P=0.028)$, and IL17 (6.9 $\pm 3.6 \mathrm{pg} / \mathrm{dL}$ vs. 0.2 $\pm 0.3 \mathrm{pg} / \mathrm{dL}, P=0.028)$ in mice recipients of $\mathrm{BMI}^{-/-}$compared with WT Tregs (Figure 6C). Moreover, histopathological analyses of the colon from mice which received the transfer of $\mathrm{BMI}^{-/}$ Tregs revealed neutrophilic inflammatory infiltration within the lamina propria of the colon and significant differences in blinded histologic scores $(12.0 \pm 0.82$ vs. $0 \pm 0.00, P=0.029$, Figure $6, \mathrm{D}$ and $\mathrm{E})$. The histology score is a numerical value from 0-26 based on the following parameters: percentage of area involved, number of follicle aggregates, edema, fibrosis, epithelial erosion/ ulceration, crypt loss, infiltration of mononuclear cells, infiltration of polymorphonuclear cells.

In conclusion, our ex vivo and in vivo experimental results suggest that BMI1-mediated repression of proinflammatory gene networks is required for the maintenance of Treg identity and colitis suppression. Therefore, we expect that disturbances in this pathway contribute to mucosal inflammation and human IBD. Not only does this have mechanistic relevance, but it also contributes to the knowledge of potential effects of novel therapeutic approaches that target BMI for other diseases, such as cancer.

\section{Discussion}

The current study was designed to extend our knowledge on one of the most evolutionarily conserved epigenomic pathways, namely Polycomb-mediated gene regulation, and its influence on the immune response that accompanies inflammatory diseases of the colon. We report the results of a new multi-faceted investigation that began with human CD lymphocytes and progressed through mechanistic in vivo genetically engineered animal and cell modeling to demonstrate an unsuspected EZH2-independent function for BMI1 in human and murine FOXP3 ${ }^{+} \mathrm{CD}^{+}$lymphocytes. A major finding is the nonredundant role for BMI1 in repression of the Th17-like pro-inflammatory gene networks in Treg cells. This function was inferred from the demonstration that, in contrast to the histone marks which are typically regulated by both PRC1 and PRC2, knockdown of BMI1 results in a genome-wide remodeling of the location of the H3K27ac marks in the human Jurkat lymphocyte. More definitive evidence supporting this observation was also derived by experiments that involved the genetic inactivation of $\mathrm{BMI} 1$ in mouse $\mathrm{FOXP}^{+} \mathrm{CD}^{+}$cells, and showed key changes in chromatin accessibility, gene transcription, and protein expression. The functional relevance of BMI1-mediated epigenomic events is reflected by the development of a pro-inflammatory phenotype in $\mathrm{Bmil}^{-/}$animals and the in vivo dysfunction of BMI1-deficient Tregs in a transfer model of colitis. Several congruent results from additive experimental systems (specifically, chromatin accessibility assays and transcriptional analyses) suggest that the inactivation of BMI1 is linked to de-repression of a Th17/Th1-like proinflammatory network. Considering that these experiments began from the analyses of de-regulated gene networks found in human $\mathrm{CD}$, the mechanistic findings reported here have strong implications to human IBD.

IBD is a complex immune-mediated disorder involving the interplay between host genetics, environmental factors, and the gut microbiota. Although current IBD therapies continue to lessen the burden of disease, recurrence and lack of response by a significant proportion of patients pose a major clinical problem. Cell therapy strategies including the transfer of in vitro derived Tregs exist, yet there is general acknowledgement of a theoretical risk of the conversion of Tregs to a proinflammatory phenotype (40). This phenomenon that we report here can be brought about by the disruption of the BMI1-mediated pathway. To gain mechanistic insight into this important problem, we investigated how epigenetic abnormalities govern $\mathrm{CD}^{+} \mathrm{T}$ cell fate decisions and colitis development.

The first notable observation derived from our mechanistic investigation is the degree to which BMI1 gene regulation appears to be independent of EZH2 enzymatic activity in lymphocytes. Indeed, the canonical function of BMI1 (PRC1) is the maintenance of EZH2 (PRC2) repressed genes through recruitment to H3K27me3 histone marks. Furthermore, we and others have described a key role for EZH2 in murine Treg biology (16, 25), and suspected that these pathways in Tregs work in complete synchrony. However, both ChIP-Seq and ATAC-Seq studies performed on EZH2- or BMI1-depleted cells support the notion that these Polycomb complexes have, at least in part, significant nonredundant functions in Tregs. For instance, while H3K27me3 levels were reduced in both cases (redundant function), BMI1 deficiency led to marked H3K27ac upregulation in human Jurkat cells and over 5,000 unique accessible chromatin regions assayed by ATAC-Seq in murine Tregs. This finding suggests that one important PRC2-independent function for PRC1 in lymphocytes might be gene activation through the remodeling of enhancers reflected by the robust mobilization of the H3K27ac mark. However, one cannot infer from our data a consistent repressor role for BMI1, as neither direct nor indirect effects of BMI1 deficiency have been definitively tested. PRC1 has recently been shown to anchor both activating enhancer-promoter loops in Drosophila (41) in addition to repressor loops previously described (42). Indeed, over two-thirds of differentially expressed genes in BMI1 deficient $\mathrm{FOXP3}^{+}$cells were downregulated when compared with wild type. Thus, these new emerging functions of the PRC1 complex in both repression and activation of genomic regulatory regions are areas of intense interest. At the time of writing, 3 active trials of BMI1-inhibition (BMI1 inhibitor PTC596) in cancer are listed on ClinicalTrials.gov, and this report raises awareness of a novel immunologic effect of this pathway in cancer therapy. We do note, however, that EZH2 inhibitors have also been used in clinical trials in the cancer arena. Some of these trials have revealed major gastrointestinal side effects including diarrhea (ClinicalTrials.gov Identifier: NCT03480646), a finding which should prompt increased awareness and monitoring for GI-related toxicities. 
Germane to cancer biology is the recent observation that RING1B drives enhancers of oncogenic programs in breast cancer through recruitment by the transcription factors ER $\alpha$ and FOXA1 (43). As we detected BMI1-RING1B protein complexes in murine Tregs and activated splenic Tregs, specific chromatin recruitment through diverse transcription factor/RING1B complexes is also likely. As Th17-relevant transcription factors such BATF, RUNX1 and ROR $\gamma \mathrm{T}$ are known regulators of chromatin structure $(39,44,45)$ this model of BMI1 recruitment to Th17 phenotypic networks is intriguing.

We also underscore our discovery that motifs for Th17-inducing transcription factors such as CTCF, ETS1, and RUNX1 were highly enriched within the open chromatin of BMI1 deficient FOXP3 $^{+}$lymphocytes. Very recently, compelling ATAC-Seq data using murine IL6-stimulated cells confirmed CTCF, potentially recruited by BATF and ETS1, to regulate enhancer/promoter looping interaction at the IL21 and IL17A/F locus further substantiating our findings that BMI1 regulates a Th17-like program within $\mathrm{FOXP3}^{+}$cells (46). Congruent with prior work that FOXP3 and BATF (Th17 pioneer factor) are among the top differentially expressed transcription factors within Crohn's mucosa-associated $\mathrm{CD} 4^{+} \mathrm{T}$ cells (25), the significance of these regulatory mechanisms to human $\mathrm{CD}$ is quite high. Indeed, conditional BMI deletion then models this mechanism of specific loss of control of Th17-lineage defining programs in human $\mathrm{CD}$-associated $\mathrm{FOXP}^{+}$cells. Treg-specific ablation of BMI1 and loss of epigenetic repression of this Th17 module of transcription factors led to a pro-inflammatory phenotype in mice and failure to control colitis in an adoptive transfer model. Thus the mechanistic details of colitis induction by BMI1-deficient Tregs is linked, at least in part, to the activation of specific immunological pathways of significant human relevance through chromatin remodeling, and in particular enhancer regions.

A limitation to this report is that the bulk of the mechanistic work was derived from peripheral or in vitro derived Treg subsets. It is reassuring that recent single cell sequencing analysis from mucosal specimens in both UC and CD identify derangements within the Treg cellular subset. However, deeper sequencing experiments need be performed from isolated $\mathrm{CD} 4^{+}$cells to directly confirm the epigenetic mechanisms identified within.

In summary, taking our results in aggregate, we propose that BMI1 plays an important role in enforcing Treg identity in vitro and in vivo. Loss of Treg identity via genetic or transient BMI1 depletion perturbs the epigenome and converts Tregs into Th1/ Th17-like proinflammatory cells, a transition relevant to human $\mathrm{CD}$-associated $\mathrm{CD} 4^{+} \mathrm{T}$ cells. This contribution is of importance as epigenetic programs are frequently misregulated in human disease, and can be also easily perturbed by small molecule inhibitors, both events that bear strong translational potential.

\section{Methods}

Mouse strains. FOXP3-CRE (B6-Tg(Foxp $\left.3^{\text {EGFP-Cre }}\right) 1 \mathrm{cJbs} / \mathrm{J}$ mice were initially purchased from the Jackson Laboratory and back-crossed to a C57BL/6 background in conventional housing conditions in the Mayo Clinic Animal Facility. The Bmi1-floxed mice (originally generated at UT Southwestern, Sean Morrison Lab, ref. 47) were provided by Raul A. Urrutia. C57BL/6J mice and C57BL/6J RAG-1 mice were initially purchased from The Jackson Laboratory and bred in conventional housing in the Mayo Clinic animal facility. The CAR transgenic mouse was obtained through the NIAID Exchange Program of the National Institutes of Health: Balb/cJ[Tg]CARdelta1[Tg]DO11.10 mouse line 4285 (48). The CAR mouse expresses the Coxsackie Adenovirus Receptor (CAR) transgene and is optimal for adenoviral transduction studies in resting lymphocytes. All of the mice used in experiments were males 6 to 21 weeks in age. The mice were cocaged, age-matched in experiments.

Isolation of murine primary $T$ cells. Murine WT and FoxP3-GFP spleens were disrupted utilizing frosted microscope slides with sterile tissue disruption buffer (DPBS with calcium and magnesium plus $0.5 \%$ FCS), and filtered through a sterile $40 \mu \mathrm{m}$ Falcon cell strainer. The cell slurry was lysed with Red Blood Cell lysis buffer, washed and resuspended with MACs buffer (DPBS without calcium and magnesium plus $0.5 \%$ BSA plus $2 \mathrm{mM}$ EDTA). The cell suspension was then filtered through a $70 \mu \mathrm{m}$ Falcon cell strainer. Murine naive $\mathrm{CD} 4^{+}$were isolated using $\mathrm{CD} 4{ }^{+} \mathrm{CD} 62 \mathrm{~L}^{+} \mathrm{T}$ Cell Isolation Kit (Miltenyi Biotec, catalog 130-106-643). The murine $\mathrm{T}$ regulatory cells were isolated using the $\mathrm{CD} 4{ }^{+} \mathrm{CD} 25^{+}$Regulatory T Cell Isolation Kit (Miltenyi Biotec, catalog 130-091-041). Per the supplied protocol, the positively selected cell fraction of $\mathrm{CD} 4^{+} \mathrm{CD} 25^{+}$cells was separated over a second column to increase purity of the Treg population $\left(\mathrm{CD} 4{ }^{+} \mathrm{CD} 25^{++}\right)$.

Isolation of human primary $C D 4^{+} C D 45 R A T$ cells. The $\mathrm{CD} 4^{+}$ $\mathrm{CD}_{4} \mathrm{RA}^{+}$cells were isolated using the human $\mathrm{CD} 4^{+} \mathrm{T}$ Cell Isolation Kit (Miltenyi Biotec, catalog 130-096-533) and CD45RA Microbeads (Miltenyi Biotec, catalog 130-045-901) according to the manufacturer's instructions. PBMCs were obtained from the Department of Pathology and Laboratory Medicine, Mayo Clinic.

Isolation of lamina propria $C D 4^{+} \mathrm{T}$ cells. Isolation of lamina propria $C D 4^{+}$lymphocytes has been previously described (49). Briefly, resection specimens or mucosal biopsies of the terminal ileum were obtained from patients with Crohn's disease and age- and sexmatched healthy control individuals. The tissue initially underwent mechanical disruption in the presence of $1 \mathrm{mM}$ EDTA in a $37^{\circ} \mathrm{C} \mathrm{CO}_{2}$ incubator for 30 minutes, followed by collagenase $(1 \mathrm{mg} / \mathrm{mL})$, DNase $(1 \mathrm{mg} / \mathrm{mL})$, and trypsin inhibitor $(1 \mathrm{mg} / \mathrm{mL})$ overnight at $4^{\circ} \mathrm{C}$ in cRPMI supplemented with $10 \%$ human serum. After passage through a $70-\mu \mathrm{m}$ cell strainer, the buffy coat was isolated using Ficoll gradient centrifugation. $\mathrm{CD}^{+}$lamina propria cells were isolated using magnetic bead sorting (CD4 ${ }^{+} \mathrm{T}$ Cell Isolation Kit, Miltenyi Biotec, catalog 130-091-155) according to the manufacturer's instructions. Purification (>95\% purity) was confirmed by flow cytometry.

Differentiation of human primary $T$ cells. Following bead isolation, naive $\mathrm{T}$ cells were cultured in a 24 -well tissue culture plate for 7 days in cRPMI media (RPMI, 10\% FCS, 10 mM HEPES, 1 mM sodium pyruvate, $1 \%$ nonessential amino acids, $2 \mathrm{mM}$ L-glutamine, penicillin/streptomycin, culture-grade $50 \mu \mathrm{M}$ 2-mercaptoethanol) with Treg-inducing cytokines: anti-CD28 (2 $\mu \mathrm{g} / \mathrm{mL}$, BD BioSciences, catalog 555725), plate-bound anti-CD3 (2 $\mu \mathrm{g} / \mathrm{mL}, \mathrm{BD}$ BioSciences, catalog 555329), IL-2 (10 ng/mL, Peprotech, catalog 200-02), and TGF- $\beta$ (5 ng/mL, Peprotech, catalog 100-21C). During the 7 day incubation, additional media with cytokines was added on day 3. On day 5 , cells were harvested and washed once with DPBS. Cells were resuspended in fresh cRPMI media with only CD28 and replated on freshly coated anti-CD3 plates until day 7 harvest for RNA and ATAC-Seq analysis. For ATAC-Seq, 100,000 cells were aliquoted and resuspended in freezing buffer $(10 \%$ DMSO, 10\% FCS, RPMI) until further processing. 
Cell cultures. Jurkat T cells (clone E6-1, ATCC, TIB-152) were maintained in medium A (RPMI 1640 supplemented with 10\% FCS, $10 \mathrm{mmol} / \mathrm{L}$ HEPES, $\mathrm{pH}$ 7.4, and $2 \mathrm{mmol} / \mathrm{L}$ L-glutamine) at less than $10^{6}$ cells $/ \mathrm{mL}$.

Cytokine analysis. Cytokine levels were determined in supernatants from cultured cells and murine serum using the BD cytometric bead array mouse Th1/Th2/Th17 kit (BD Bioscience, catalog 560485) according to the instructions of the manufacturer and analyzed using FCAP Array version 3 software (Soft Flow Hungary Ltd).

Immunoblotting. Primary murine T cells or Jurkat cells were harvested, washed with cold, PBS, and lysed with RIPA lysis buffer containing 50 mmol/L Tris-HCL, pH 7.4, 1\% NP-40, 150 mmol/L NaCl, and $2 \mathrm{mmol} / \mathrm{L}$ EDTA. Protein was detected by Western blot using the indicated primary antibodies.

Antibodies for immunoblotting analysis. Primary antibodies used were rabbit monoclonal anti-Bmi1 (1:1000, Cell Signaling Technology, catalog 5756), mouse monoclonal anti- $\beta$-actin (1:1000, Cell Signaling Technology, catalog 3700), goat monoclonal anti-Mel18 (1:250, Abcam, catalog ab5267), rabbit monoclonal anti-H2Aub (1:1500, Cell Signaling Technology, catalog 8240), mouse monoclonal anti-H2A (1:1000, Cell Signaling Technology, catalog 3636).

RNA isolation, cDNA synthesis, and quantitative real-time PCR. Per previously published methods (25), total RNA was isolated using the RNeasy Mini Kit (QIAGEN, catalog 74104) per manufacturer protocol. cDNA was synthesized from $0.5-1 \mu \mathrm{g}$ of total RNA with random primers using the SuperScript Kit III First-Strand (Invitrogen, Thermo Fisher Scientific). Reverse transcription products $(2 \mu \mathrm{L})$ was used for each real-time PCR. PCRs were performed in $20 \mu \mathrm{L}$ of total volume that contained primers and $10 \mu \mathrm{L}$ Express SYBR Green ER Quantitative PCR Supermaster Mix (Invitrogen, Thermo Fisher Scientific).

Targeted microarray. Per previously published methods (25), CD4 ${ }^{+}$ cells were first isolated from the spleens of 7- to 8-week-old mice using a Miltenyi magnet per manufacturer protocol (refer to Isolation of Primary Murine T cells in the Methods section). $\mathrm{CD}^{+} \mathrm{T}$ cells were then subjected to sorting for Foxp3 $\mathrm{GFP}^{+}$cells using an Aria FACS sorter. The 15,00040,000 cells collected were then stored in RNAprotect cell reagent (QIAGEN, catalog 76526) and later processed using the RNAeasy Micro kit (QIAGEN, catalog 74004) for RNA isolation. Transcript levels were compared between $\mathrm{BMI}^{-/-}(n=5)$ or $\mathrm{BMI}^{+/+}$Tregs $(n=5)$ in 2 separate batches utilizing SABioscience pathway-focused mouse Th cell differentiation PCR arrays. PCR cycles were completed, and SYBR was detected with the Bio-Rad CFX96 real-time PCR machine.

$T$ cell transfer model of chronic colitis. Chronic colitis was induced via adoptive transfer of naive $C D 4{ }^{+} \mathrm{CD} 45 \mathrm{RB}^{\text {hi }} \mathrm{T}$ cells derived from WT donors using our previously published method. Briefly, spleens were removed from donor WT $\mathrm{C} 57 \mathrm{BL} / 6 \mathrm{~J}$ mice and macerated between the frosted ends of 2 microscopy glass slides to produce single-cell suspensions in PBS supplemented with $0.1 \%$ FBS. The CD $4^{+}$ $T$ cells were enriched by negative selection using magnetic beads (see Isolation of primary $T$ cells). The enriched $\mathrm{T}$ cells were labeled with anti-CD45RB and anti-CD25 (catalogs 103308 and 10217, respectively; BioLegend) and sorted for CD $45 \mathrm{RB}^{\text {hi }} \mathrm{CD} 25^{\text {lo }}$ with a FACS Aria (FACS; BD Biosciences). GFP ${ }^{+}$Treg cells were sorted from the spleens of either WT or BMI1-KO mice. We injected $5 \times 10^{5} \mathrm{CD}_{4} \mathrm{RB}^{\text {hi }}$ cells and $1.5 \times 10^{5} \mathrm{GFP}^{+}$cells suspended in $200 \mu \mathrm{L}$ sterile PBS intraperitoneally into C57BL6J RAG-1-KO animals. The animals were switched to nonirradiated chow, and their weight was recorded weekly until sacrificed at 9 weeks. The histologic disease activity was assessed by our participating gastrointestinal pathologist who was blinded to the study grouping. DAI was assessed by laboratory personnel that were blinded to the study conditions. DAI was determined by a score that included stool consistency, hunching posture, degree of wasting, and colonic stiffness.

PLA and confocal microscopy of fixed cells. The proximity ligation method assesses protein-protein interactions $(50,51)$. This protocol was adapted from previously published methods from our laboratory (24). Images were processed using the Zen lite 2012 software (Carl Zeiss).

Antibodies for PLA. Primary antibodies used were rabbit monoclonal anti-RING1B (Cell Signaling Technology, catalog 5694), mouse monoclonal anti-BMI1 (Invitrogen, Thermo Fisher Scientific, catalog MA5-15919; 1:100-1:250).

Flow cytometry. For cell-surface staining, cells were directly stained with the indicated antibodies in stain buffer (BioLegend). For intracellular staining, cells were treated as indicated, fixed and permeabilized with buffer (BioLegend True-Nuclear Transcription Factor Buffer Set, catalog 424401) and stained with relevant primary antibodies and fluorochrome-conjugated secondary antibodies. Antibodies used were anti-mouse FOXP3 Alexa Fluor (AF) 647 (BioLegend, catalog 320014), anti-rat IgG AF647 (BioLegend, catalog 400526), anti-mouse CD44 PE/Cy7 (BioLegend, catalog 104418), anti-Armenian hamster IgG PE/Cy7 (BioLegend, catalog 400921), anti-human IL-2 AF647 (BioLegend, catalog 500315), anti-mouse CD62L PE/Cy7 (BioLegend, catalog 104418). Cells were subsequently subjected to flow cytometry for FACS and electronically gated on live cells and single cells for analysis.

Transfection of Jurkat cells. Jurkat T cells were transiently transfected with indicated plasmids by electroporation at $315 \mathrm{mV}$ for $10 \mathrm{~ms}$ and harvested 36 to 48 hours later. Jurkat cells $\left(2 \times 10^{6}\right)$ were transfected with 120 nM of ON-TARGETplus Human BMI1 siRNA (648) (Dharmacon, catalog L-005230-01-0005) or 100 nM of ON-TARGETplus Human EZH2 siRNA (2146) (Dharmacon, catalog L-004218-00-0005).

ChIP coupled with deep sequencing (ChIP-Seq). Cultured Jurkat cells were harvested from suspension and pelleted down. ChIP protocol used from previously published methods and in collaboration with referenced authors (52). Antibodies used: anti-H3K27ac (Abcam, catalog ab4729), anti-H3K27me3 (Cell Signaling Technology, catalog 9733S). ChIP DNA was purified using the Mini-Elute PCR purification kit (QIAGEN) after treatment with DNase-free RNase A $(10 \mathrm{mg} / \mathrm{mL}$, Thermo Fisher Scientific, catalog EN0531) and Proteinase K (20 mg/ $\mathrm{mL}$, Ambion, catalog AM2546). Purified DNA was subjected to targeted real-time PCR to confirm enrichment. ChIP DNA library preparation was performed using $10 \mathrm{ng}$ per reaction, and input DNA using the Ovation Ultralow DR Multiplex System (NuGen). The ChIP-seq libraries were subjected to sequencing by the Mayo Clinic Medical Genomics Core. These were sequenced to 50 base pairs from both ends using the Illumina HiSeq 2000.

Assay for transposase-accessible chromatin by sequencing (ATAC-seq). ATAC-seq was performed from 50,000 cells following the published protocol (53). Amplified libraries were purified, and the sizes of library DNA were determined by the Fragment Analyzer (Advanced Analytical Technologies) using the High Sensitivity NGS Fragment Analysis Kit (catalog DNF-486). The enrichment of accessible region was determined by the fold difference between positive and negative genomic loci using real-time PCR. The libraries were sequenced to 51 base pairs 
from both ends on an Illumina HiSeq 4000 instrument in the Mayo Clinic Center for Individualized Medicine Medical Genomics Facility.

Bioinformatics analyses. For microarray analysis, the raw expression microarray data in.cel format (mouse MTA-1.0) were imported into the Transcriptome Analysis Console (TAC4.0, Thermo Fisher Scientific). RMA algorithm was used to normalize and summarize gene level expression level. Limma was used for differential expression where genes with fold change greater than 1.5 [initial batch ( 2 vs 2 samples) used as representative of the total replicates] and $p$ value less than 0.05 were selected as differentially expressed genes (DEGs). Average occupancy profiles of H3K27ac (Figure 4E), enrichment at the peaks of interest were evaluated using computeMatrix function in DEEPTOOLS (54). Significance was calculated using Tukey's test for multiple comparisons.

The pathway analysis on murine Treg differentially regulated genes was performed using the Gene Ontology database (GO Biological Processes 2018) in the online Enrichr tool $(55,56) \cdot \log _{10} P$ value was used to establish significance.

For ATACseq, PRC1 complex members were compiled from previous reports (57). Paired-end reads from ATAC and ChIP-seq data sets were mapped to the hg38 reference genome using BOWTIE2/2.3.3.1 (58). SAMTOOLS/1.6 (59) was used to generate and sort bam files. Bigwigs were generated by DEEPTOOLS/3.3.0 (60). Peak calling and summit identification was performed using MACS2/2.1.2 (61) with a broad cutoff of 0.05 for histone marks and with turning the shifting model option off. Heatmaps and occupancy average profiles were generated using computeMatrix and plotHeatmap tools included in DEEPTOOLS/3.3.0. Hierarchal clustering of ATAC regions was performed on merged ATAC peaks in all conditions using seqminer/1.3.4. Kmeans linear was used for clustering normalization. ATAC regions of interest were characterized by running the ReMAP algorithm (62), which uses publicly available genome localization data to predict significantly enriched factors based on comparison between observed overlap and predicted overlap by chance.

ScRNA seq enrichment analysis. Sixty-two DEGs detected upon Bmi1 deletion in murine Tregs $(\mathrm{FC}>2$, padj $>0.05)$ were compared with a previously published CD single cell RNA-seq data set (38). Data with at least 500 unique molecular identifiers (UMIs), log10 genes per UMI $>0.8,>250$ genes per cell and a mitochondrial ratio of less than $0.2 \%$ were extracted, normalized and integrated using the seurat package version 3.0 in $\mathrm{R}$ 4.0.2. The expression of BMI1-regulated genes was compared between Tregs (marked by FOXP3 ${ }^{+}, \mathrm{CD}^{+}$, IL2RA ${ }^{+}$) detected in GIMATS-positive and -negative specimens. Statistical differences were determined using unpaired $t$ test.
Statistics. Statistical analyses were performed with GraphPad Software version 8 (GraphPad Software). Nonparametric unpaired $t$ test was performed using Mann-Whitney $U$ test and a $P$ less than 0.05 was considered statistically significant. Data are presented as mean \pm SD or \pm SEM (as indicated in each figure legend). The GEO accession number for microarray and next-generation sequencing data is GSE171190.

Study approval. All animal work was done in accordance with and reviewed/approved by the Mayo Clinic IACUC, protocol A00001268-16-R19.

\section{Author contributions}

MMG contributed to conception, planning, design and execution of experiments, data acquisition, analysis and interpretation, and manuscript preparation. AOB contributed the design of experiments, data analysis and interpretation, manuscript preparation and critical revisions. PAS contributed to conducting experiments with data acquisition and manuscript revision. MRS contributed to conducting experiments with data acquisition and interpretation, manuscript revision. TCS provided data analysis (histology). $\mathrm{FHH}$ and JMG contributed to data interpretation, analysis, and review of manuscript. GPR and HRG contributed to data interpretation and manuscript review. ZS, ZY, and AN contributed to data analysis and interpretation. MBB contributed to data interpretation. AQW contributed to data interpretation and manuscript review. $\mathrm{AM}$ contributed to data acquisition and interpretation. SJ contributed to data interpretation, and critical review of manuscript. RU contributed to the study concept and design, data interpretation, and critical manuscript revisions with important intellectual content. WAF provided study supervision, contributed to study concept and design, data analysis and interpretation, manuscript preparation and critical revisions, and also acquired funding.

\section{Acknowledgments}

We would like to thank the Microscopy and Cell Analysis Core for their support with flow cytometric analysis and cell sorting, as well as the Mayo Clinic Animal Care facility for the ongoing care of animals used in our research. This work was supported by the NIH (grant R01AI089 714-022) and NIDDK (grants T32DK007198 and P30DK084567).

Address correspondence to: William A. Faubion Jr., 200 First Street SW, Rochester, Minnesotta 55905, USA. Phone: 507.293.0970; Email: faubion.william@mayo.edu.
1. Smids $\mathrm{C}$, et al. Intestinal T cell profiling in inflammatory bowel disease: linking $\mathrm{T}$ cell subsets to disease activity and disease course. JCrohns Colitis. 2018;12(4):465-475.

2. Reinecker HC, et al. Enhanced secretion of tumour necrosis factor-alpha, IL-6, and IL-1 beta by isolated lamina propria mononuclear cells from patients with ulcerative colitis and Crohn's disease. Clin Exp Immunol. 1993;94(1):174-181.

3. Ye M, et al. Deletion of IL-6 exacerbates colitis and induces systemic inflammation in IL-10-deficient mice. JCrohns Colitis. 2020;14(6):831-840.

4. Kim JM, et al. Regulatory T cells prevent catastrophic autoimmunity throughout the lifespan of mice. Nat Immunol. 2007;8(2):191-197.

5. Zhou X, et al. Instability of the transcription factor Foxp3 leads to the generation of pathogenic memory T cells in vivo. Nat Immunol. 2009;10(9):1000-1007.

6. Ishikawa $\mathrm{D}$, et al. Tregs are dysfunctional in vivo in a spontaneous murine model of Crohn's disease. Mucosal Immunol. 2013;6(2):267-275.

7. Hegazy AN, et al. Circulating and tissue-resident $\mathrm{CD}^{+} \mathrm{T}$ cells with reactivity to intestinal microbiota are abundant in healthy individuals and function is altered during inflammation. Gastroenterology. 2017;153(5):1320-1337.

8. Britton GJ, et al. Microbiotas from humans with inflammatory bowel disease alter the balance of gut Th17 and ROR $\mathrm{t}^{+}$regulatory T cells and exacerbate colitis in mice. Immunity. 2019;50(1):212-224.

9. Sakaguchi S, et al. Immunologic self-tolerance maintained by activated T cells expressing IL-2 receptor alpha-chains (CD25). Breakdown of a single mechanism of self-tolerance causes various autoimmune diseases. Jimmunol. 1995;155(3):1151-1164.

10. Sakaguchi S. Naturally arising Foxp3-expressing CD25+CD4+ regulatory T cells in immunological tolerance to self and non-self. Nat Immunol. $2005 ; 6(4): 345-352$.

11. Fontenot JD, et al. Foxp3 programs the develop- 
ment and function of $\mathrm{CD} 4+\mathrm{CD} 25+$ regulatory $\mathrm{T}$ cells. Nat Immunol. 2003;4(4):330-336.

12. Zhou L, et al. TGF-beta-induced Foxp 3 inhibits $\mathrm{T}(\mathrm{H}) 17$ cell differentiation by antagonizing RORgammat function. Nature. 2008;453(7192):236-240.

13. Ohkura N, et al. Development and maintenance of regulatory T cells. Immunity. 2013;38(3):414-423.

14. Bennett CL, et al. The immune dysregulation, polyendocrinopathy, enteropathy, X-linked syndrome (IPEX) is caused by mutations of FOXP3. Nat Genet. 2001;27(1):20-21.

15. Georgiev P, et al. Regulatory T cells: the many faces of foxp3. JClin Immunol. 2019;39(7):623-640.

16. DuPage $M$, et al. The chromatin-modifying enzyme Ezh2 is critical for the maintenance of regulatory $\mathrm{T}$ cell identity after activation. Immunity. 2015;42(2):227-238.

17. Cavalli G, Heard E. Advances in epigenetics link genetics to the environment and disease. Nature. 2019;571(7766):489-499.

18. Portela A, Esteller M. Epigenetic modifications and human disease. Nat Biotechnol. 2010;28(10):1057-1068.

19. Tumes DJ, et al. The polycomb protein Ezh2 regulates differentiation and plasticity of $\mathrm{CD} 4(+)$ Thelper type 1 and type 2 cells. Immunity. 2013;39(5):819-832.

20. Yang XP, et al. EZH2 is crucial for both differentiation of regulatory $\mathrm{T}$ cells and $\mathrm{T}$ effector cell expansion. Sci Rep. 2015;5:10643.

21. Raaphorst FM, et al. Distinct BMI-1 and EZH2 expression patterns in thymocytes and mature $\mathrm{T}$ cells suggest a role for Polycomb genes in human $\mathrm{T}$ cell differentiation. J Immunol. 2001;166(10):5925-5934.

22. Wei G, et al. Global mapping of H3K4me3 and $\mathrm{H} 3 \mathrm{~K} 27 \mathrm{me} 3$ reveals specificity and plasticity in lineage fate determination of differentiating CD4+ T cells. Immunity. 2009;30(1):155-167.

23. Antignano F, Zaph C. Regulation of CD4 T-cell differentiation and inflammation by repressive histone methylation. Immunol Cell Biol. 2015;93(3):245-252.

24. Bamidele AO, et al. Disruption of FOXP3-EZH2 interaction represents a pathobiological mechanism in intestinal inflammation. Cell Mol Gastroenterol Hepatol. 2019;7(1):55-71.

25. Sarmento OF, et al. The role of the histone methyltransferase enhancer of zeste homolog 2 (EZH2) in the pathobiological mechanisms underlying inflammatory bowel disease (IBD). J Biol Chem. 2017;292(2):706-722.

26. Zhou J, et al. Targeting EZH2 histone methyltransferase activity alleviates experimental intestinal inflammation. Nat Commun. 2019;10(1):2427.

27. Laugesen A, et al. Molecular mechanisms directing PRC2 recruitment and H3K27 methylation. Mol Cell. 2019;74(1):8-18.
28. Hojfeldt JW, et al. Accurate H3K27 methylation can be established de novo by SUZ12-directed PRC2. Nat Struct Mol Biol. 2018;25(3):225-232.

29. Senthilkumar R, Mishra RK. Novel motifs distinguish multiple homologues of Polycomb in vertebrates: expansion and diversification of the epigenetic toolkit. BMC Genomics. 2009;10:549.

30. Gray F, et al. BMI1 regulates PRC1 architecture and activity through homo- and hetero-oligomerization. Nat Commun. 2016;7:13343.

31. Liu X, et al. BMI1 and MEL18 promote colitisassociated cancer in mice via REG3B and STAT3. Gastroenterology. 2017;153(6):1607-1620.

32. Blackledge NP, et al. Targeting Polycomb systems to regulate gene expression: modifications to a complex story. Nat Rev Mol Cell Biol. 2015;16(11):643-649.

33. Goswami S, et al. Modulation of EZH2 expression in T cells improves efficacy of anti-CTLA- 4 therapy. J Clin Invest. 2018;128(9):3813-3818.

34. Lu C, et al. Histone H3K36 mutations promote sarcomagenesis through altered histone methylation landscape. Science. 2016;352(6287):844-849.

35. Gao Z, et al. PCGF homologs, CBX proteins, and RYBP define functionally distinct PRC1 family complexes. Mol Cell. 2012;45(3):344-356.

36. Gil J, O'Loghlen A. PRC1 complex diversity: where is it taking us? Trends Cell Biol. 2014;24(11):632-641.

37. Kreso A, et al. Self-renewal as a therapeutic target in human colorectal cancer. Nat Med. 2014;20(1):29-36.

38. Martin JC, et al. Single-cell analysis of Crohn's disease lesions identifies a pathogenic cellular module associated with resistance to anti-TNF therapy. Cell. 2019;178(6):1493-1508.

39. Pham D, et al. Batf pioneers the reorganization of chromatin in developing effector $\mathrm{T}$ cells via Ets1-dependent recruitment of Ctcf. Cell Rep. 2019;29(5):1203-1220.

40. Canavan JB, et al. Developing in vitro expanded CD45RA+ regulatory T cells as an adoptive cell therapy for Crohn's disease. Gut. 2016;65(4):584-594.

41. Loubiere $\mathrm{V}$, et al. Widespread activation of developmental gene expression characterized by PRC1-dependent chromatin looping. Sci $A d v$. 2020;6(2):eaax4001.

42. Ogiyama $\mathrm{Y}$, et al. Polycomb-dependent chromatin looping contributes to gene silencing during drosophila development. Mol Cell.2018;71(1):73-88.

43. Chan HL, et al. Polycomb complexes associate with enhancers and promote oncogenic transcriptional programs in cancer through multiple mechanisms. Nat Commun. 2018;9(1):3377.

44. Karwacz K, et al. Critical role of IRF1 and BATF in forming chromatin landscape during type 1 regulatory cell differentiation. Nat Immunol. 2017;18(4):412-421.
45. Rodriguez-Caparros A, et al. Notch signaling controls transcription via the recruitment of RUNX1 and MYB to enhancers during T cell development. J Immunol. 2019;202(8):2460-2472.

46. Weaver CT, et al. Th17: an effector CD4 T cell lineage with regulatory $\mathrm{T}$ cell ties. Immunity. 2006;24(6):677-688.

47. Mich JK, et al. Prospective identification of functionally distinct stem cells and neurosphere-initiating cells in adult mouse forebrain. Elife. 2014;3:e02669.

48. Wan YY, et al. Transgenic expression of the coxsackie/adenovirus receptor enables adenoviral-mediated gene delivery in naive T cells. Proc Natl Acad Sci U S A. 2000;97(25):13784-13789.

49. Rahman MK, et al. The pathogen recognition receptor NOD2 regulates human FOXP3+ T cell survival. J Immunol. 2010;184(12):7247-7256.

50. Alam MS. Proximity ligation assay (PLA). Curr Protoc Immunol. 2018;123(1):e58.

51. Bagchi S, et al. In situ proximity ligation assay (PLA). Methods Mol Biol. 2015;1318:149-159.

52. Lomberk G, et al. Distinct epigenetic landscapes underlie the pathobiology of pancreatic cancer subtypes. Nat Commun. 2018;9(1):1978.

53. Buenrostro JD, et al. Transposition of native chromatin for fast and sensitive epigenomic profiling of open chromatin, DNA-binding proteins and nucleosome position. Nat Methods. 2013;10(12):1213-1218.

54. Ramirez F, et al. deepTools2: a next generation web server for deep-sequencing data analysis. Nucleic Acids Res. 2016;44(w1):W160-W165.

55. Chen EY, et al. Enrichr: interactive and collaborative HTML5 gene list enrichment analysis tool. BMC Bioinformatics. 2013;14:128.

56. Kuleshov MV, et al. Enrichr: a comprehensive gene set enrichment analysis web server 2016 update. Nucleic Acids Res. 2016;44(W1):W90-W97.

57. Abdouh M, et al. The Polycomb repressive complex 1 protein BMI 1 is required for constitutive heterochromatin formation and silencing in mammalian somatic cells. J Biol Chem. 2016;291(1):182-197.

58. Langmead B, Salzberg SL. Fast gappedread alignment with Bowtie 2. Nat Methods. 2012;9(4):357-359.

59. Li H, et al. The sequence alignment/map format and SAMtools. Bioinformatics. 2009;25(16):2078-2079.

60. Ramirez F, et al. deepTools: a flexible platform for exploring deep-sequencing data. Nucleic Acids Res. 2014;42(W1):W187-W191.

61. Zhang Y, et al. Model-based analysis of ChIP-Seq (MACS). Genome Biol. 2008;9(9):R137.

62. Cheneby J, et al. ReMap 2018: an updated atlas of regulatory regions from an integrative analysis of DNA-binding ChIP-seq experiments. Nucleic Acids Res. 2018;46(D1):D267-D275. 